HU-EP-04/44

hep-th/0408169

\title{
Mirror symmetry for topological sigma models with generalized Kähler geometry
}

\author{
Stefano Chiantese, Florian Gmeiner and Claus Jeschek \\ Humboldt Universität zu Berlin, \\ Institut für Physik, \\ Newtonstrasse 15, 12489 Berlin, Germany, \\ Email: chiantes, gmeiner, jeschek@physik.hu-berlin.de.
}

\begin{abstract}
We consider topological sigma models with generalized Kähler target spaces. The mirror map is constructed explicitly for a special class of target spaces and the topological A and B model are shown to be mirror pairs in the sense that the observables, the instantons and the anomalies are mapped to each other. We also apply the construction to open topological models and show that A branes are mapped to B branes. Furthermore, we demonstrate a relation between the field strength on the brane and a two-vector on the mirror manifold.
\end{abstract}




\section{Introduction}

Since the work of Witten [1] two dimensional topological field theories have been considered to study mirror symmetry. The aim of this paper is to construct explicitly the mirror map for topological models with a certain class of target spaces having a generalized geometry in the sense of Hitchin [2].

Two dimensional nonlinear sigma models describe maps $\phi: \Sigma \rightarrow X$ from the Riemann surface to the target space. Supersymmetry on the Riemann surface constraints the target space geometry. This link between supersymmetry and geometry becomes especially interesting for the $\mathcal{N}=(2,2)$ sigma model. In [3] it was realized that the most general target space geometry is a bi-Hermitian geometry described in terms of the following data $\left(g, I_{+}, I_{-}, H\right)$. The Riemannian metric $g$ is Hermitian with respect to two different complex structures $I_{+}$and $I_{-}$for right and left movers, and the $H$-flux is a closed 3-form which can be expressed locally as the field strength of a $B$-field. It was found recently [4] that this geometry can be described equivalently by a (twisted) generalized Kähler structure $(\mathrm{GKS})$ if $(H \neq 0) H=0$. It is defined by two commuting generalized complex structures $(\mathrm{GCS}) \mathcal{J}_{1 / 2}$, which are endomorphisms of $T \oplus T^{*}$, the combined tangent and cotangent spaces of the target space. In the following we will restrict ourselves to $H=0$.

The $\mathcal{N}=(2,2)$ sigma model can be twisted in two different ways. The twist consists in changing the spin of the fermions with the vector/axial symmetry so that one obtains a bosonic theory. Twisting with the vector (axial) current, one gets the topological A (B) model. The twist allows to define supersymmetry also on a curved Riemann surface with the advantage that one can use a localization principle.

To incorporate mirror symmetry along the lines of [5] into the generalized geometry picture we follow [6, 7]. Work in this direction has also been done in [8].

In the first part of this paper we will show how one can define a mirror map $\mathcal{M}$ for a specific class of target spaces and how it acts on the GKS. In section 3 we verify this using the language of spinor lines. We apply these results to the generalized topological sigma models [9, 10] described above. We confirm that mirror symmetry 
transforms a generalized A model with specific target space to a generalized B model on the mirror dual target. It is checked explicitly in section 4 how the data of the sigma models (observables, instantons and $U(1)$ anomalies) transform under $\mathcal{M}$ and how they behave in the limit of only one complex structure. The generalized framework can also be applied to open topological theories [9, 11. In the last part of this paper we check how mirror symmetry acts on the gluing conditions which define A and B branes. We see explicitly how A and B branes on different target spaces are related by $\mathcal{M}$. Furthermore, we see that the two-form field is mapped to a two-vector, which is related to a noncommutative structure on the mirror manifold. In the appendix we give details about the construction of the generalized topological sigma models.

\section{The mirror map $\mathcal{M}$}

In this section we take the GKS to be defined on a specific 6-manifold [7] (see also [6]). Since our interest is only to show how certain properties of the two GCS behave under a specific bundle isomorphism, called mirror symmetry, we do not need more general 6-manifolds.

Generically, the two GCS of a GKS are given in the $T \oplus T^{*}$ basis by

$$
\mathcal{J}_{1 / 2}=\frac{1}{2}\left(\begin{array}{cc}
I_{+} \pm I_{-} & -\left(\omega_{+}^{-1} \mp \omega_{-}^{-1}\right) \\
\omega_{+} \mp \omega_{-} & -\left(I_{+}^{T} \pm I_{-}^{T}\right)
\end{array}\right),
$$

where the complex structures $I_{+}$and $I_{-}$are independent sections $\left(\forall p \in M^{6}\right)$ in the twistor space $\mathcal{Z} M^{6}$. Note that we always assume integrability for the two complex structures. We can also define a generalized metric by $G=-\mathcal{J}_{1} \mathcal{J}_{2}$.

Suppose that we take a trivial fibre bundle $M^{6}=T^{6}$ with fibre $F=T^{3}$ over the

base space $B=T^{3}$, thus $M^{6}=T^{3} \oplus T^{3}$. Therefore we have the following splitting of the generalized tangent space:

$$
\left(T \oplus T^{*}\right) \otimes \mathbb{C}=\left(T_{B} \oplus T_{F} \oplus T_{B}^{*} \oplus T_{F}^{*}\right) \otimes \mathbb{C}
$$

This choice is for computational convenience, but one can consider a more general $M^{6}$ as a nontrivial $T^{3}$ torus fibration over a general three dimensional base space 
without changing the essence of our argument [5]. Furthermore, we want to consider only GCS which are adapted in the sense of [7], i.e.

$$
\mathcal{J}_{1 / 2}: T_{F} \oplus T_{F}^{*} \rightarrow T_{B} \oplus T_{B}^{*}
$$

Respecting additionally the algebraic properties of GCS we take

$$
\begin{gathered}
I_{+} \pm I_{-}=\left(\begin{array}{ll}
\tilde{I}_{+} \pm \tilde{I}_{-} & -\left(\tilde{I}_{+} \pm \tilde{I}_{-}\right)
\end{array}\right), \\
\omega_{+} \mp \omega_{-}=\left(\begin{array}{cc}
\tilde{\omega}_{+} \mp \tilde{\omega}_{-} & -\left(\tilde{\omega}_{+} \mp \tilde{\omega}_{-}\right)
\end{array}\right) .
\end{gathered}
$$

Note that $\tilde{I}_{+}, \tilde{I}_{-}$and $\tilde{\omega}_{+}, \tilde{\omega}_{-}$are not complex structures and Kähler forms, respectively. Note also that to satisfy the properties $I_{ \pm}^{2}=-1$ and $\omega_{ \pm}^{T}=-\omega_{ \pm}^{T}$ one has to require $\tilde{I}_{ \pm}^{2}=1$ and $\tilde{\omega}_{ \pm}^{T}=\tilde{\omega}_{ \pm}$.

We are now prepared to write the specific GCS:

$$
\mathcal{J}_{1 / 2}=\frac{1}{2}\left(\begin{array}{cccc} 
& -\left(\tilde{I}_{+} \pm \tilde{I}_{-}\right) & & -\left(\tilde{\omega}_{+}^{-1} \mp \tilde{\omega}_{-}^{-1}\right) \\
\tilde{I}_{+} \pm \tilde{I}_{-} & & \tilde{\omega}_{+}^{-1} \mp \tilde{\omega}_{-}^{-1} & \\
& -\left(\tilde{\omega}_{+} \mp \tilde{\omega}_{-}\right) & \left.\tilde{I}_{+}^{T} \pm \tilde{I}_{-}^{T}\right)
\end{array}\right),
$$

where the transpose and inverse operation only indicate that the indices are up/down, appropriately.

We have choosen the specific manifold not by accident. We know from [5] that mirror symmetry can be considered as a T-duality transformation along the $T^{3}$-fibre over a 3-dimensional base space. We adopt this idea and conjecture the mirror map $\mathcal{M}$ to be a map which acts on the generalized tangent bundle $\left(T \oplus T^{*}\right) \otimes \mathbb{C}$ as an bundle isomorphism [7, 6]. Moreover, this isomorphism should have the property of an involution, $\mathcal{M}^{2}=1$. The mirror map in the generalized tangent space induces naturally a map for the GKS, i.e., we get mirror transformed GCS $\hat{\mathcal{J}}_{1 / 2}$ and hence a mirror transformed generalized metric $\hat{G}$.

Let us define the mirror map such that it acts as an identity on $T_{B}, T_{B}^{*}$ and as a "flip" on $T_{F} \leftrightarrow T_{F}^{*}$ :

$$
\mathcal{M}: T_{B} \oplus T_{F} \oplus T_{B}^{*} \oplus T_{F}^{*} \rightarrow T_{B} \oplus T_{F}^{*} \oplus T_{B}^{*} \oplus T_{F},
$$


explicitly

$$
\mathcal{M}=\left(\begin{array}{llll}
1 & & & \\
& & & 1 \\
& & 1 & \\
& 1 & &
\end{array}\right) .
$$

We get a conjugated GCS in the following way:

$$
\hat{\mathcal{J}}_{1 / 2}=\mathcal{M} \circ \mathcal{J}_{1 / 2} \circ \mathcal{M}^{-1}: T_{B} \oplus T_{F}^{*} \oplus T_{B}^{*} \oplus T_{F} \rightarrow T_{B} \oplus T_{F}^{*} \oplus T_{B}^{*} \oplus T_{F} .
$$

Applying this construction explicitly we get

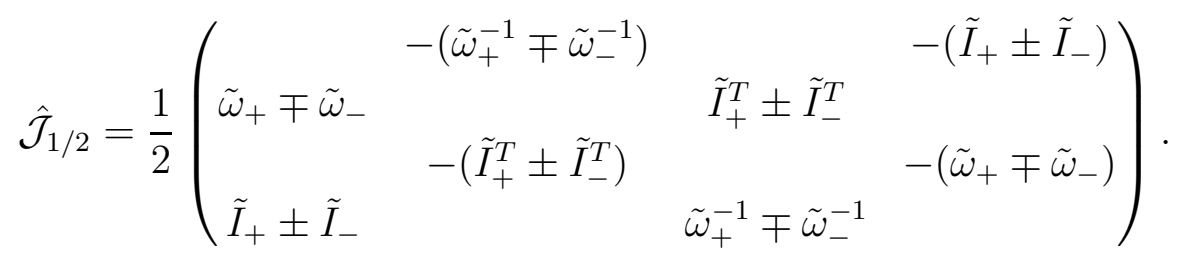

To compare $\hat{\mathcal{J}}_{1 / 2}$ with $\mathcal{J}_{1 / 2}$ we reinterpret $\hat{\mathcal{J}}_{1 / 2}$ as a map $T_{B} \oplus T_{F} \oplus T_{B}^{*} \oplus T_{F}^{*} \rightarrow$ $T_{B} \oplus T_{F} \oplus T_{B}^{*} \oplus T_{F}^{*}$ instead of (9). We then use the fiber metric $g_{F}$ and its inverse and we write them back into $\hat{\mathcal{J}}_{1 / 2}$ (see also [8]). By using the identity $\omega=g I$, we get finally

$$
\hat{\mathcal{J}}_{1 / 2}=\frac{1}{2}\left(\begin{array}{cccc} 
& -\left(\tilde{I}_{+} \mp \tilde{I}_{-}\right) & & -\left(\tilde{\omega}_{+}^{-1} \pm \tilde{\omega}_{-}^{-1}\right) \\
& & \tilde{\omega}_{+}^{-1} \pm \tilde{\omega}_{-}^{-1} & \\
\tilde{\omega}_{+} \pm \tilde{\omega}_{-} & -\left(\tilde{\omega}_{+} \pm \tilde{\omega}_{-}\right) & \left.\tilde{I}_{+}^{T} \mp \tilde{I}_{-}^{T}\right)
\end{array}\right),
$$

where now $\hat{\mathcal{J}}_{1 / 2}$ are again maps

$$
\hat{\mathcal{J}}_{1 / 2}: T_{B} \oplus T_{F} \oplus T_{B}^{*} \oplus T_{F}^{*} \rightarrow T_{B} \oplus T_{F} \oplus T_{B}^{*} \oplus T_{F}^{*} .
$$

This is the mirror transformed complex structure. In the following we will denote by $\mathcal{M}$ the mirror map, which is the combined operation of $\mathcal{M}$ and the reinterpretation of maps. We see immediatly that mirror symmetry interchanges the two GCS:

$$
\begin{array}{ccc}
\mathcal{J}_{1 / 2} & \longleftrightarrow & \hat{\mathcal{J}}_{1 / 2}=\mathcal{J}_{2 / 1} \\
\left(I_{+}, I_{-}\right) & \longleftrightarrow & \left(\hat{I}_{+}, \hat{I}_{-}\right)=\left(I_{+},-I_{-}\right) .
\end{array}
$$

When $M^{6}$ is a nontrivial torus fibration, using the same remark above, also the mirror manifold $\hat{M}^{6}$ is a nontrivial torus fibration. 
Additionally, we will derive this result by means of maximal isotropics, associated to pure spinor lines [2, 4]. But to achieve this it is usefull to remember first the splitting of $\left(T \oplus T^{*}\right) \otimes \mathbb{C}$ into subbundles with respect to the GCS $\mathcal{J}_{1 / 2}$.

\section{Pure spinors, maximal isotropics and the mirror map}

Let us assume we have a generic GKS on a 6-manifold $M^{6}$. With the two given commuting (integrable) GCS, $\mathcal{J}_{1 / 2}$, we get a decomposition of $\left(T \oplus T^{*}\right) \otimes \mathbb{C}$ into a direct sum of four subbundles, as it is shown in [4]. We want to review this decomposition.

On the one hand this can be understood by firstly noting that the generalized metric $G$ gives a decomposition into two subbundles of dimension $3_{\mathbb{C}}$ each. This can be done explicitly by using the projectors $P_{ \pm}=\frac{1}{2}(1 \pm G)$, where the associated subbundles having eigenvalues \pm 1 and carrying a positive/negative definite metric are called $C_{ \pm} \otimes$ $\mathbb{C}$

$$
\left(T \oplus T^{*}\right) \otimes \mathbb{C}=\left(C_{+} \oplus C_{-}\right) \otimes \mathbb{C}
$$

It can be shown that elements of $C_{ \pm} \otimes \mathbb{C}$ can be written as $(B=0)$

$$
C_{+} \otimes \mathbb{C}=\{X+g X \mid X \in T \otimes \mathbb{C}\}, \quad C_{-} \otimes \mathbb{C}=\{X-g X \mid X \in T \otimes \mathbb{C}\},
$$

where the generalized metric $G$ is purely Riemannian,

$$
G=\left(\begin{array}{ll} 
& g^{-1} \\
g &
\end{array}\right) .
$$

On the other hand, since the GCS commute with $G$, we can also decompose the generalized tangent bundle with respect to the GCS $\mathcal{J}_{1 / 2}$. This we will do by the useful formulae,

$$
\begin{aligned}
\mathcal{J}_{1} & =\left.\pi\right|_{C_{+}} ^{-1} I_{+} \pi P_{+}+\left.\pi\right|_{C_{-}} ^{-1} I_{-} \pi P_{-}, \\
\mathcal{J}_{2} & =\left.\pi\right|_{C_{+}} ^{-1} I_{+} \pi P_{+}-\left.\pi\right|_{C_{-}} ^{-1} I_{-} \pi P_{-},
\end{aligned}
$$

where $\pi: C_{ \pm} \rightarrow T$ is a projection.

We will denote the $i$ eigenbundle of $\mathcal{J}_{1 / 2}$, or equivalently the graphs of the maps $-i \mathcal{J}_{1 / 2}$, by $L_{1 / 2}$, respectively

$$
\begin{aligned}
& L_{1}=\left\{X+g X \mid X \in T_{+}^{1,0}\right\} \oplus\left\{X-g X \mid X \in T_{-}^{1,0}\right\}, \\
& L_{2}=\left\{X+g X \mid X \in T_{+}^{1,0}\right\} \oplus\left\{X-g X \mid X \in T_{-}^{0,1}\right\} .
\end{aligned}
$$


The generalized tangent bundle decomposes therefore in

$$
\left(T \oplus T^{*}\right) \otimes \mathbb{C}=L_{1} \oplus \overline{L_{1}}=L_{2} \oplus \overline{L_{2}}
$$

Since the two GCS commute we can decompose $L_{1 / 2}$ further by $\mathcal{J}_{2 / 1}$. We indicate \pm for the eigenvalues $\pm i$ corresponding to the second splitting, e.g.

$$
L_{1} \oplus \overline{L_{1}}=L_{1}^{+} \oplus L_{1}^{-} \oplus \overline{L_{1}^{+}} \oplus \overline{L_{1}^{-}}
$$

where

$$
\begin{array}{ll}
L_{1}^{+}=\left\{X+g X \mid X \in T_{+}^{1,0}\right\}, & L_{1}^{-}=\left\{X-g X \mid X \in T_{-}^{1,0}\right\}, \\
L_{2}^{+}=\left\{X+g X \mid X \in T_{+}^{1,0}\right\}, & L_{2}^{-}=\left\{X-g X \mid X \in T_{-}^{0,1}\right\} .
\end{array}
$$

We see that $L_{2}=L_{1}^{+} \oplus \overline{L_{1}^{-}}$and

$$
C_{ \pm} \otimes \mathbb{C}=L_{1 / 2}^{ \pm} \oplus \overline{L_{1 / 2}^{ \pm}}
$$

These careful observations make clear that by changing $I_{-} \rightarrow-I_{-}$we do not affect the $C_{+}$-bundle and moreover only interchange in the $C_{-}$-bundle holomorphic with antiholomorphic objects with respect to $I_{-}$. Thus, mirror symmetry interchanges the subbundles $L_{1}^{-} \leftrightarrow \overline{L_{1}^{-}}$.

Now we are prepared to come back to the question: How does the mirror map look like for pure spinor lines? We will attack this question with our specifications for the 6-manifold given in section 2. For the theory of spinor lines the reader should consult 4. Firstly, we conjecture this map and apply it to the generating pure spinor lines. Let us remember that pure spinor lines single out maximal isotropics. More precisely, the (involutive) maximal isotropics of our interest are $L_{1}^{+}, L_{1}^{-}$. These can be described by the following four pure spinor lines $\phi_{i}, i \in\{1, \ldots, 4\}$

$$
\begin{array}{ll}
0=L_{1}^{+} \cdot \phi_{1}=L_{1}^{+} \cdot \Omega_{+}^{(3,0)}, & 0=L_{1}^{-} \cdot \phi_{2}=L_{1}^{-} \cdot \Omega_{-}^{(3,0)}, \\
0=L_{1}^{+} \cdot \phi_{3}=L_{1}^{+} \cdot e^{i \omega_{+}}, & 0=L_{1}^{-} \cdot \phi_{4}=L_{1}^{-} \cdot e^{-i \omega_{-}},
\end{array}
$$

where $\Omega_{ \pm}^{(3,0)} \in \Lambda^{\text {od }}$ are holomorphic top degree forms with respect to $I_{+}, I_{-}$and $\omega_{ \pm} \in$ $\Lambda^{e v}$ are the Kähler forms. The operation '.' means Clifford multiplication: $(X+\xi) \cdot \phi=$ $X\lrcorner \phi+\xi \wedge \phi$, where $X \in T, \xi \in T^{*}, \phi \in \Lambda^{\bullet}$. 
In what follows we choose an appropriate local trivialization for our forms, i.e. local complex coordinates with respect to either $I_{+}$or $I_{-}$. We split them into an imaginary part $y^{i}, i \in\{1,2,3\}$, and a real part $x^{\alpha}, \alpha \in\{1,2,3\}$, which are the coordinates in the base and the fibre, respectively. Thus, e.g.

$$
\begin{aligned}
e^{i \omega_{+}} & =1+i d x^{i} d y^{i}+d x^{12} d y^{12}+d x^{23} d y^{23}+d x^{13} d y^{13}+i d x^{123} d y^{123} \\
\Omega_{+}^{(3,0)} & =\left(d x^{1}+i d y^{1}\right) \wedge\left(d x^{2}+i d y^{2}\right) \wedge\left(d x^{3}+i d y^{3}\right) .
\end{aligned}
$$

We conjecture the mirror map acting on pure spinor lines by

$$
\begin{aligned}
\mathcal{M}: \Lambda^{\text {ev/od }} & \rightarrow \Lambda^{\text {od/ev }} \\
\phi & \rightarrow\left(\partial_{X_{3}}+d x^{3}\right) \cdot\left(\partial_{X_{2}}+d x^{2}\right) \cdot\left(\partial_{X_{1}}+d x^{1}\right) \cdot \phi,
\end{aligned}
$$

where $T_{F}=\operatorname{span}\left\{\partial_{X_{\alpha}}\right\}, T_{F}^{*}=\operatorname{span}\left\{d x^{\alpha}\right\}$ and $\phi \in \Lambda^{\bullet}$.

Using the property that $\left.\partial_{X_{\alpha}}\right\lrcorner d x^{\beta}=\delta_{\alpha}{ }^{\beta}$, we apply the mirror map to our pure spinor lines $\phi_{i}$ to get

$$
\begin{array}{lrrrrr}
\hat{\phi}_{1}= & \mathcal{M} \cdot \Omega_{+}^{(3,0)}= & e^{i \omega_{+}}, & \hat{\phi}_{2}= & \mathcal{M} \cdot \Omega_{-}^{(3,0)}= & e^{i \omega_{-}} \\
\hat{\phi}_{3}= & \mathcal{M} \cdot e^{i \omega_{+}}= & -\Omega_{+}^{(3,0)}, & \hat{\phi}_{4}= & \mathcal{M} \cdot e^{-i \omega_{-}}= & -\overline{\Omega_{-}^{(3,0)}} .
\end{array}
$$

Let us now focus on the maximal isotropics which are associated to these mirror transformed pure spinor lines $\hat{\phi}_{i}, i \in\{1, \ldots, 4\}$. We see immediately that $L_{1}^{+}$is untouched by the map $\mathcal{M}$ but in the $C_{-}$-bundle it interchanged $L_{1}^{-}$with $\overline{L_{1}^{-}}$. Thus, we verified exactly our previous result.

\section{Generalized topological sigma models}

Topological sigma models in the generalized framework can be defined as usual by twisting the fermionic spin with the vectorial/axial $U(1)$ charge. The crucial difference is given by the fact that we allow for two different complex structures. For details on the definition of the models see appendix $\mathrm{A}$.

We want to write the BRST operators in the $T \oplus T^{*}$ bundle. Let us define the fermionic basis

$$
\psi:=\left(\psi_{+}+\psi_{-}\right) \in T, \quad \rho:=g\left(\psi_{+}-\psi_{-}\right) \in T^{*}, \quad \Psi:=\left(\begin{array}{c}
\psi \\
\rho
\end{array}\right)
$$


where $\psi_{ \pm}$are elements of $\pi C_{ \pm}$. Then the BRST operators of the generalized B and A model take the form 9

$$
\begin{aligned}
& Q_{B}=\left\langle\left(\begin{array}{c}
\partial_{1} \phi \\
g \partial_{0} \phi
\end{array}\right),\left(1+i \mathcal{J}_{1}\right) \Psi\right\rangle, \\
& Q_{A}=\left\langle\left(\begin{array}{c}
\partial_{1} \phi \\
g \partial_{0} \phi
\end{array}\right),\left(1+i \mathcal{J}_{2}\right) \Psi\right\rangle,
\end{aligned}
$$

where $<,>$ is the natural metric on $T \oplus T^{*}$ [2, 4].

The classical $U(1)_{A / V}$ symmetry can be broken by quantum effects. This anomaly is given in terms of the first Chern class of the $L_{1 / 2}$ bundle for the $B / A$ model [10]. The cancellation of this anomaly constrains the target space geometry via $c_{1}\left(L_{1 / 2}\right)=0$.

Now we want to show how the relevant quantities of the generalized B model with the target space $M^{6}$ are mapped to the ones of the generalized A model ${ }^{1}$ with the mirror target space $\hat{M}^{6}$. From section 2 we know that $\mathcal{M}: \mathcal{J}_{1} \rightarrow \mathcal{J}_{2}$ so that $\mathcal{M}$ : $Q_{B} \rightarrow Q_{A}$. We also know that $\left(I_{+}, I_{-}\right)$is mapped to $\left(I_{+},-I_{-}\right)$under the mirror map and equation (21) tells us that $\mathcal{M}: L_{1} \rightarrow L_{2}$. Therefore, $\mathcal{M}: c_{1}\left(L_{1}\right) \rightarrow c_{1}\left(L_{2}\right)$ and the anomaly cancellation of the generalized B model gets mapped to that of the generalized A model.

The next step is to show that the observables of generalized B and A model are mirrors of each other. We show this for the local observables of the closed topological sector, but first let us remember how they were constructed in [9]. Following [1], one has to construct scalar BRST invariant field configurations. Writing the BRST variations in the $T \oplus T^{*}$ bundle, we get $^{2}$

$$
\delta_{B / A} \Phi=\Psi_{1 / 2}:=\frac{1}{2}\left(1+i \mathcal{J}_{1 / 2}\right) \Psi \in \bar{L}_{1 / 2}, \quad \Phi:=\left(\begin{array}{c}
\phi \\
g \phi
\end{array}\right) .
$$

The nilpotency properties $\delta_{B / A}^{2}=0$ of the BRST variations then yield $\delta_{B / A} \Psi_{1 / 2}=0$. Thus, $\Psi_{1 / 2}$ are the configurations we are looking for in the generalized B/A model.

\footnotetext{
${ }^{1}$ This choice is of course arbitrary, one could as well start with the generalized A model on $M^{6}$ and map it to the B model on $\hat{M}^{6}$

${ }^{2}$ This $\Phi$ is an element of $T \oplus T^{*}$ and should not be confused with the chiral superfield defined in appendix A
} 
The space of observables is then given by

$$
\left(\mathcal{O}_{f}\right)_{B / A}=f_{a_{1} \cdots a_{n}}(\phi) \Psi_{1 / 2}^{a_{1}} \cdots \Psi_{1 / 2}^{a_{n}}
$$

which can be mapped to the exterior algebra bundle $\Lambda^{k} \bar{L}_{1 / 2}^{*} \simeq \Lambda^{k} L_{1 / 2}$ since $f$ is skew symmetric in the indices $a$. Performing the BRST variation of $\left(\mathcal{O}_{f}\right)_{B / A}$, one realizes that the map is actually an isomorphism,

$$
\left\{Q_{B / A},\left(\mathcal{O}_{f}\right)_{B / A}\right\}=\left(\mathcal{O}_{d_{\bar{L}_{1 / 2}} f}\right)_{B / A}
$$

where $d_{\bar{L}_{1 / 2}}=\partial_{\bar{L}_{1 / 2}}^{+}+\partial_{\bar{L}_{1 / 2}}^{-}$is the Lie algebroid derivative such that $d_{\bar{L}_{1 / 2}}: C^{\infty}\left(\Lambda^{k} L_{1 / 2}\right) \rightarrow$ $C^{\infty}\left(\Lambda^{k+1} L_{1 / 2}\right)$ [4. Since $\mathcal{M}: \bar{L}_{1} \rightarrow \bar{L}_{2}$, the cohomologies of the differential complexes for the generalized A and B models are mirror pairs.

We want to do the same for the generalized instantons [9]. The instantons are the fixed points of the BRST transformations. Performing the Wick rotation $\partial_{0} \phi \rightarrow i \partial_{2} \phi$ on the Riemann surface, one gets the instanton equations

$$
\delta_{B / A} \Psi=\left(1-i \mathcal{J}_{1 / 2}\right)\left(\begin{array}{c}
i \partial_{2} \phi \\
g \partial_{1} \phi
\end{array}\right)=0 .
$$

These equations tell us that the instantons of the generalized B model are mapped to those of the generalized A model under the mirror map.

To conclude this section we want to show that the mirror map between the generalized topological models gives the old (in the sense of Witten [1]) A/B model with target space $\hat{M}^{6}$ as a mirror of the old B/A model with target space $M^{6}$. It is only necessary to note the following. The generalized $\mathrm{B}$ model yields the old $\mathrm{B}$ and $\mathrm{A}$ models under the identifications $I_{+}=I_{-}$and $I_{+}=-I_{-}$respectively. Under the same identifications the generalized A model yields the old A and B model. To give just one example, the constant maps $\phi: \Sigma \rightarrow M^{6}$ of the old B model are mapped to the holomorphic maps $\phi: \Sigma \rightarrow \hat{M}^{6}$ of the old A model.

\section{Topological branes and their mirrors}

In this section we want to investigate how topological branes behave under the mirror map $\mathcal{M}$. We will strongly follow the notation and conventions used in [1] and references therein. 
Branes in the topological A/B model (A/B branes) can be defined by a gluing matrix $R: T \rightarrow T$, which encodes information about the mapping of left- and rightmoving fermions at the boundary $\partial \Sigma[12,13]$. The gluing conditions read

$$
\psi_{-}=R \psi_{+}
$$

In the generalized picture this translates to [11]

$$
\mathcal{R}: T \oplus T^{*} \rightarrow T \oplus T^{*}, \quad \mathcal{R} \Psi=\Psi,
$$

where $\Psi$ is defined in (30). $\mathcal{R}$ respects the natural metric $<\cdot, \cdot>$ on $T \oplus T^{*}$, squares to one, i.e. $\mathcal{R}^{2}=1$, and anticommutes with $G$, i.e. $G \mathcal{R}+\mathcal{R} G=0$.

In the (physical) gluing framework the operator $\mathcal{R}$ contains the information about Dirichlet and Neumann boundary conditions (bc). It defines a smooth distribution $\mathcal{D} \subset T$ which has rank equal to the dimension of the brane. In case of an integrable distribution we even have (Frobenius) a maximal integral submanifold $\mathcal{D}$.

From a different point of view, the above properties of $\mathcal{R}$ serve to consider the projection operator $\frac{1}{2}(1+\mathcal{R})$ to define a special almost Dirac structure $\tau_{\mathcal{D}}^{0}$ (a real, maximal isotropic sub-bundle),

$$
\tau_{\mathcal{D}}^{0}=T \mathcal{D} \oplus \operatorname{Ann}(T \mathcal{D}) \subset T \oplus T^{*},
$$

which is (Courant) integrable iff $\mathcal{D}$ is integrable.

The extension of $\mathcal{R}$ by a closed two-form $F \in \Omega^{2}(\mathcal{D}), d F=0$, on the submanifold $\mathcal{D}$ corresponds to [1]

$$
\left.\tau_{\mathcal{D}}^{F}=\left\{\frac{1}{2}(1+\mathcal{R})(X+\xi)=(X+\xi):\left.(X+\xi) \in T \mathcal{D} \oplus T^{*} M\right|_{\mathcal{D}},\left.\xi\right|_{\mathcal{D}}=X\right\lrcorner F\right\}
$$

and is equivalent to the definition of a generalized tangent bundle given in [4. This gluing matrix is given by

$$
\mathcal{R}=\left(\begin{array}{ll}
1 & \\
F & 1
\end{array}\right)\left(\begin{array}{cc}
r & \\
& -r^{t}
\end{array}\right)\left(\begin{array}{cc}
1 & \\
-F & 1
\end{array}\right)=\left(\begin{array}{ccc}
r & \\
F r+r^{t} F & -r^{t}
\end{array}\right),
$$

where $r$ is an operator which carries the gluing information for the fermions (see also [12, 13]). 
Let us focus on the A/B branes in the corresponding A/B model. This means that the $U(1)$ currents $j_{ \pm}=\omega_{ \pm}\left(\psi_{ \pm}, \psi_{ \pm}\right), \psi_{ \pm} \in T$, have to fulfill the matching conditions

$$
0=j_{+} \pm j_{-}=\frac{1}{2}\left\langle\Psi, \mathcal{J}_{2 / 1} \Psi\right\rangle
$$

for the A/B model, respectively.

Moreover, combining this with the gluing conditions for the fermions, we obtain a stability condition for $\mathcal{R}$, or equivalently, a stability condition for $\tau_{\mathcal{D}}^{F}$. Using also $\{G, \mathcal{R}\}=0$, one gets:

$$
\begin{aligned}
& \text { A branes: } \mathcal{R}_{\mathcal{J}}=-\mathcal{J}_{1} \mathcal{R} \text { and } \mathcal{R J}_{2}=\mathcal{J}_{2} \mathcal{R} \\
& \text { B branes: } \mathcal{R} \mathcal{J}_{1}=\mathcal{J}_{1} \mathcal{R} \quad \text { and } \quad \mathcal{R J}_{2}=-\mathcal{J}_{2} \mathcal{R} \text {. }
\end{aligned}
$$

We will call the (anti)commuting constraints $\mp$-stability with respect to a certain GCS. Thus, the A/B model is $\mathcal{J}_{1 / 2}^{-}$stable and additionally $\mathcal{J}_{2 / 1}^{+}$stable. This reflects the fact that the generalized tangent bundle $\tau_{\mathcal{D}}^{F}$ in the $\mathrm{A} / \mathrm{B}$ model splits into $\pm i$ eigenbundles of $\mathcal{J}_{2 / 1}$ or, in other words, it becomes a stable subbundle of $L_{2 / 1} \oplus \overline{L_{2 / 1}}$, respectively:

$$
\text { A/B model: } \quad \tau_{\mathcal{D}}^{F}=\tau_{\mathcal{D}}^{F+} \oplus \tau_{\mathcal{D}}^{F-}, \quad \text { w.r.t. } \quad L_{2 / 1} \oplus \overline{L_{2 / 1}}
$$

We are now prepared to apply the mirror map $\mathcal{M}$ for the structures we just introduced. Therefore, the gluing operator $\mathcal{R}$ gets mapped to $\hat{\mathcal{R}}=\mathcal{M} \mathcal{R} \mathcal{M}^{-1}$ and one can show that the properties for $\hat{\mathcal{R}}$ are the same as they were for $\mathcal{R}$. As before, we take $M^{6}$ which has a $T^{3}$ fibration, then mirror symmetry interchanges Neumann bc with Dirichlet bc in the fibre ${ }^{3}$. Remember that under mirror symmetry the GCS (also the A/B model) get interchanged. Therefore it is easy to see that the $U(1)$ current conditions get mapped to each other and A/B branes get naturally mapped to B/A branes. But note that on the mirror side the stability conditions are formulated with $\hat{\mathcal{R}}$.

Furthermore, in case of non-vanishing $F \in \Omega^{2}(\mathcal{D})$, let us focus on the part of (40), where only $F$ appears. Then we obtain the following symbolical shape of $\hat{\mathcal{R}}$

$$
\mathcal{R}=\left(\begin{array}{cc}
r & \\
\square & -r^{t}
\end{array}\right) \quad \longrightarrow \quad \hat{\mathcal{R}}=\left(\begin{array}{cc}
\hat{r} & \square \\
& -\hat{r}^{t}
\end{array}\right)
$$

\footnotetext{
${ }^{3}$ The mirror map $\mathcal{M}$ is only a special case of the more general T-duality transformation and therefore this statement can be extended.
} 
with a bi-vector $\beta=F^{-1}$ in the upper triangular part. Thinking of $F$ in components this means that the indices get raised. We will denote this by

$$
\tau_{\hat{\mathcal{D}}}^{\beta}=\left\{\frac{1}{2}(1+\hat{\mathcal{R}})(X+\xi)=(X+\xi):\left.(X+\xi) \in T \hat{M}\right|_{\hat{\mathcal{D}}} \oplus N^{*} \hat{\mathcal{D}},\left.X\right|_{N \hat{\mathcal{D}}}=\beta(\xi)\right\}
$$

where $N^{*} \hat{\mathcal{D}}$ is the conormal bundle to $\hat{\mathcal{D}}$. For example, if we start with a brane which has only a worldvolume in the fibre directions and a non-vanishing two-form $F$, it will be mapped to a brane which corresponds to a "point". But on the mirror side $F$ disappears and we find a bi-vector $\beta$ in the fibre directions instead. This can be interpreted as a noncommutative deformation of $\hat{M}$, as has been argued in [9].

This brings us immediately to the proposal to investigate the case of having at the same time both independent structures, a two-form and a bi-vector. This would correspond to $B$ and $\beta$ transformations in the sense of [4] and therefore we get a natural extension of the generalized tangent bundle. Until now we worked in the classical regime only. It would be very interesting to shed more light (maybe along the lines of [9]) on the $\alpha^{\prime}$ corrections that appear in the full string theory. A connection to [14] should appear in this context.

\section{Acknowledgements}

We would like to thank H. Dorn and C. Sieg for interesting discussions, F. Witt for mathematical support and I. Runkel for proofreading the paper.

The work of the authors is supported by a Graduiertenkolleg grant of the DFG (The Standard Model of Particle Physics - structure, precision tests and extensions).

\section{A Appendix}

In this appendix we formulate the topological sigma models in the generalized formalism. We start with the nonlinear sigma model formulated in $\mathcal{N}=(1,1)$ superfield form:

$$
S=\frac{1}{2} \int \mathrm{d}^{2} \sigma \mathrm{d}^{2} \theta(g+B)\left(D_{+} \Phi, D_{-} \Phi\right), \quad \text { where }
$$




$$
D_{ \pm}=\frac{\partial}{\partial \theta^{ \pm}}+i \theta^{ \pm} \partial_{ \pm} \quad ; \quad \partial_{ \pm}:=\partial_{0} \pm \partial_{1}
$$

The $\mathcal{N}=(1,1)$ SUSY transformations are generated by $Q_{ \pm}$, defined as

$$
Q_{ \pm}^{(1)}:=\frac{\partial}{\partial \theta^{ \pm}}-i \theta^{ \pm} \partial_{ \pm}
$$

and the chiral superfield can be expanded in components as

$$
\Phi=\phi+\theta^{+} \psi_{+}+\theta^{-} \psi_{-}+\theta^{-} \theta^{+} F
$$

An additional supersymmetry can be defined by 3 ]

$$
Q_{ \pm}^{(2)}:=I_{ \pm} D_{ \pm}
$$

This is a well defined $(1,1)$ supersymmetry, if $I_{ \pm}$is a pair of integrable almost complex structures on $T$ and $g$ is Hermitian with respect to both $I_{+}$and $I_{-}$. Furthermore the almost complex structures have to be covariantly constant w.r.t. covariant derivatives with connection:

$$
\Gamma_{ \pm b c}^{a}:=\Gamma_{b c}^{a} \pm g^{a d} H_{d b c}
$$

where $\Gamma$ is the Levi-Civita connection. We get the following relation between the two connections

$$
\Gamma_{+b c}^{a} \psi_{+}^{b} \psi_{-}^{c}=-\Gamma_{-b c}^{a} \psi_{-}^{b} \psi_{+}^{c}
$$

The variations of the superfield (49) can be written in components as

$$
\begin{aligned}
\delta_{+}^{(1)} \phi & =\psi_{+} & \delta_{-}^{(1)} \phi & =\psi_{-} \\
\delta_{+}^{(1)} \psi_{+} & =-i \partial_{+} \phi & \delta_{-}^{(1)} \psi_{+} & =F \\
\delta_{+}^{(1)} \psi_{-} & =-F & \delta_{-}^{(1)} \psi_{-} & =-i \partial_{-} \phi \\
\delta_{+}^{(2)} \phi & =I_{+} \psi_{+} & \delta_{-}^{(2)} \phi & =I_{-} \psi_{-} \\
\delta_{+}^{(2)} \psi_{+} & =i I_{+} \partial_{+} \phi & \delta_{-}^{(2)} \psi_{+} & =I_{-} F \\
\delta_{+}^{(2)} \psi_{-} & =-I_{+} F & \delta_{-}^{(2)} \psi_{-} & =i I_{-} \partial_{-} \phi
\end{aligned}
$$

We can integrate out the auxiliary field $F$ using the equations of motion

$$
F^{a}=\Gamma_{+b c}^{a} \psi_{+}^{b} \psi_{-}^{c}
$$


Furthermore, we define combinations of the supersymmetry generators

$$
\begin{array}{lll}
Q_{+}=\frac{1}{2}\left(Q_{+}^{(1)}-i Q_{+}^{(2)}\right), & \bar{Q}_{+}=\frac{1}{2}\left(Q_{+}^{(1)}+i Q_{+}^{(2)}\right), \\
Q_{-}=\frac{1}{2}\left(Q_{-}^{(1)}-i Q_{-}^{(2)}\right), & \bar{Q}_{-}=\frac{1}{2}\left(Q_{-}^{(1)}+i Q_{-}^{(2)}\right) .
\end{array}
$$

With these we make contact to the definitions of [15].

We are now ready to define the generalized topological A(B) model. We twist the spin of the fermionic fields with the vector (axial) $U(1)$ current. The charges of the fields are given in the following table,

\begin{tabular}{l|rrrrr} 
& $q_{V}$ & $q_{A}$ & $J$ & $J_{A}$ & $J_{B}$ \\
\hline $\mathcal{P}_{+} \psi_{+}$ & -1 & -1 & $-\frac{1}{2}$ & -1 & -1 \\
$\overline{\mathcal{P}}_{+} \psi_{+}$ & +1 & +1 & $-\frac{1}{2}$ & 0 & 0 \\
$\mathcal{P}_{-} \psi_{-}$ & -1 & +1 & $+\frac{1}{2}$ & 0 & +1 \\
$\overline{\mathcal{P}}_{-} \psi_{-}$ & +1 & -1 & $+\frac{1}{2}$ & +1 & 0
\end{tabular}

where $q_{V / A}$ indicate the vector/axial charge. $J$ and $J_{A / B}=J+q_{V / A} / 2$ define the spins before and after the twist and we used projectors on the (anti-)holomorphic parts of the fields with respect to $I_{ \pm}$

$$
\mathcal{P}_{ \pm}=\frac{1}{2}\left(1-i I_{ \pm}\right), \quad \overline{\mathcal{P}}_{ \pm}=\frac{1}{2}\left(1+i I_{ \pm}\right) .
$$

As BRST operators for the generalized A and B model we take ${ }^{4}$

$$
Q_{A}:=\bar{Q}_{+}+Q_{-}, \quad Q_{B}:=\bar{Q}_{+}+\bar{Q}_{-},
$$

which act on the scalar fields of the twisted models like

$$
\begin{aligned}
\delta_{A} \phi & =\overline{\mathcal{P}}_{+} \psi_{+}+\mathcal{P}_{-} \psi_{-} & \delta_{B} \phi & =\overline{\mathcal{P}}_{+} \psi_{+}+\overline{\mathcal{P}}_{-} \psi_{-} \\
\delta_{A} \overline{\mathcal{P}}_{+} \psi_{+} & =\Gamma_{+} \overline{\mathcal{P}}_{+} \psi_{+} \mathcal{P}_{-} \psi_{-} & \delta_{B} \overline{\mathcal{P}}_{+} \psi_{+} & =\Gamma_{+} \overline{\mathcal{P}}_{+} \psi_{+} \overline{\mathcal{P}}_{-} \psi_{-} \\
\delta_{A} \mathcal{P}_{-} \psi_{-} & =\Gamma_{-} \mathcal{P}_{-} \psi_{-} \overline{\mathcal{P}}_{+} \psi_{+} & \delta_{B} \overline{\mathcal{P}}_{-} \psi_{-} & =\Gamma_{-} \overline{\mathcal{P}}_{-} \psi_{-} \overline{\mathcal{P}}_{+} \psi_{+}
\end{aligned}
$$

We can reformulate these variations in terms of generalized fields, written in the $T \oplus T^{*}$ basis, where the terms involving the connections vanish

$$
\delta_{A} \frac{1}{2}\left(1+i \mathcal{J}_{1}\right) \Psi=0, \quad \delta_{B} \frac{1}{2}\left(1+i \mathcal{J}_{2}\right) \Psi=0,
$$

where the definition of $\Psi$ can be found in (30).

\footnotetext{
${ }^{4}$ Note that [1] uses a different definition for the world sheet fermions, which leads to a different BRST operator for the A model, $Q_{A}=Q_{+}+\bar{Q}_{-}$.
} 


\section{References}

[1] E. Witten, "Mirror manifolds and topological field theory," in: Mirror symmetry I, ed. S. T. Yau, AMS (1998) 121-160, hep-th/9112056.

[2] N. Hitchin, "Generalized Calabi-Yau manifolds," Q. J. Math. 54 no.3 (2003) 281-308, math.dg/0209099.

[3] S. J. Gates, C. M. Hull, and M. Rocek, "Twisted multiplets and new supersymmetric nonlinear sigma models," Nucl. Phys. B248 (1984) 157.

[4] M. Gualtieri, Generalized complex geometry. PhD thesis, Oxford University, 2004. math.dg/0401221.

[5] A. Strominger, S.-T. Yau, and E. Zaslow, "Mirror symmetry is T-duality," Nucl. Phys. B479 (1996) 243-259, hep-th/9606040.

[6] C. Jeschek, "Generalized Calabi-Yau structures and mirror symmetry," hep-th/0406046.

[7] O. Ben-Bassat, "Mirror symmetry and generalized complex manifolds," math.ag/0405303.

[8] S. Fidanza, R. Minasian, and A. Tomasiello, "Mirror symmetric SU(3)-structure manifolds with NS fluxes," hep-th/0311122

[9] A. Kapustin, "Topological strings on noncommutative manifolds," hep-th/0310057.

[10] A. Kapustin and Y. Li, "Topological sigma-models with H-flux and twisted generalized complex manifolds," hep-th/0407249.

[11] M. Zabzine, "Geometry of D-branes for general $\mathrm{N}=(2,2)$ sigma models," hep-th/0405240.

[12] C. Albertsson, U. Lindstrom, and M. Zabzine, " $\mathrm{N}=1$ supersymmetric sigma model with boundaries, I," Commun. Math. Phys. 233 (2003) 403-421, hep-th/0111161. 
[13] C. Albertsson, U. Lindstrom, and M. Zabzine, " $\mathrm{N}=1$ supersymmetric sigma model with boundaries, II," Nucl. Phys. B678 (2004) 295-316, hep-th/0202069.

[14] N. Seiberg and E. Witten, "String theory and noncommutative geometry," JHEP 09 (1999) 032, hep-th/9908142.

[15] K. Hori, S. Katz, A. Klemm, R. Pandharipande, R. Thomas, C. Vafa, R. Vakil, and E. Zaslow, Mirror Symmetry. AMS (Clay Mathematics Monographs, V. 1), 2003. 